\title{
Mécanismes moléculaires de la vision des couleurs et du daltonisme
}

Une équipe de Stanford [I,2] vient d'élucider de façon magistrale la génétique moléculaire de la vision des couleurs ainsi que celle de ses anomalies conduisant aux diverses variétés de daltonisme. Ces résultats ont fait également l'objet d'éditoriaux dans Science [3] et dans Nature [4].

Les pigments visuels sont des molécules qui absorbent la lumière. Ils sont formés d'une apoprotéine, qui porte le nom d'opsine, et d'un cofacteur dérivé de la vitamine $\mathrm{A}$, le II-cis-rétinal. Ces pigments sont localisés dans la membrane plasmique des cellules photoréceptrices. On connaît quatre pigments visuels, spécifiés par la longueur d'onde de leur maximum d'absorption. Trois d'entre eux servent à la vision colorée, absorbant à 420 nanomètres (sensible au bleu), $530 \mathrm{~nm}$ (sensible au vert) et $560 \mathrm{~nm}$ (sensible au rouge). Tous trois sont localisés dans les cônes de la rétine, un cône donné ne possédant qu'un seul pigment. Le quatrième, qui absorbe à $495 \mathrm{~nm}$, est la rhodopsine, qui permet la vision crépusculaire; il siège dans les bâtonnets, et est de loin le plus abondant.

La caractérisation des gènes de la vision des couleurs repose sur l'exploitation d'une idée qui n'est plus nouvelle, mais qui s'est avérée d'une extrême fécondité: on a d'abord pensé que la reconnaissance d'un acide nucléique par un autre (au moyen de l'hybridation) exigeait leur identité presque complète, et l'on insistait sur la notion de spécificité. On s'est aperçu ensuite que la possibilité d'hybridation entre deux molécules dépend des conditions expérimentales de l'hybridation et des lavages qui la suivent : à conditions très rigoureuses (on dit stringentes), par exemple à température élevée, hybridation très sélective; à conditions moins rigoureuses, hybridation moins sélective, par ne seraient homologues qu'à $50 \%$. Partant de cette idée, on fait l'hypothèse qu'une famille de protéines dont le rôle est similaire dérive d'un ancêtre commun, que leurs gènes ont gardé une structure voisine, et qu'à partir d'un des gènes on doit parvenir à isoler tous les autres. Comment appliquer cette hypothèse aux pigments visuels? Une protéine était mieux connue que les autres, la rhodopsine. Nathans et coll. $[I]$ ont préparé des clones d'ADN complémentaire et génomique de rhodopsine de bœuf, puis les ont utilisés pour cribler une banque d'ADN germinal humain réalisée incidemment à partir d'un des auteurs. Dans un premier temps ils ont obtenu uniquement des clones du gène de la rhodopsine humaine. Travaillant ensuite à des températures plus basses, ils isolèrent des clones provenant d'autres gènes; ils en ont établi la séquence et déduit celle des acides aminés correspondants. Ces clones étaient de deux types : le premier avait la même structure à cinq exons et le même nombre d'acides aminés que la rhodopsine (348) avec $42 \%$ d'identité. Par des expériences d'hybridations cellulaires somatiques homme-souris, ce gène a été localisé sur le chromosome 7 ( 7 q 22-7 qter); il n'est donc pas lié à l'X et a été identifié comme le gène du pigment "bleu». Par la même méthode, le gène de la rhodopsine a été localisé sur le chromosome 3 (3 q 2 I-3 qter).

Le deuxième type de clones et les sondes qui en dérivent peuvent être attribués par hybridation somatique à la partie distale du bras long du chromosome $\mathrm{X}$ (Xq 22-q 28), au voisinage du locus de la glucose 6 phosphate deshydrogénase, en accord avec les données génétiques classiques sur la transmission du daltonisme. La structure générale reste la même que celle de la rhodopsine; notamment tous ces pigments possèdent une lysine qui fixe le rétinal par une liaison covalente. Il existe cependant une différence: la séquence des nouveaux gènes montre six exons; les exons 2 à 6 correspondent aux exons I à 5 de la rhodopsine et du pigment bleu, mais il y a en $5^{\prime}$ un exon I supplémentaire. En outre, la carte de restriction permet de distinguer deux gènes différents malgré une homologie à $96 \%$, codant respectivement pour les pigments "vert" et "rouge". On peut extraire de la rétine des ARN messagers qui reconnaissent chacune de ces sondes.

Les auteurs ont alors fait une découverte inattendue; s'il existe toujours un, et un seul, gène rouge, le nombre de gènes verts, même chez les sujets à vision normale, varie de un à trois. Comment expliquer cette variabilité d'un seul des deux gènes? L'hypothèse est que ces deux gènes, qui ne sont distants que de cinq kilobases, sont disposés en tandem tête-queue (figure I), résultat sans doute d'une duplication génique au cours de l'évolution. La très grande ressemblance des deux gènes permet une recombinaison non équationnelle (voir médecine/sciences $n^{\circ} 4$, vol. I, p.214), donnant naissance à un chromosome à deux gènes et à un autre qui n'en porte aucun. Pour interpréter le fait que le gène rouge n'est jamais impliqué dans l'échange inégal, il faut admettre que ce gène est situé à la lisière de la zone de recombinaison.

Anomalies de la vision des couleurs. En pratique, elles portent sur la vision du vert et du rouge, en liaison avec le chromosome $\mathrm{X}$; celles de la vision du bleu, transmises comme un caractère autosomique récessif, restent exceptionnelles. Seuls les sujets du sexe masculin sont donc atteints. On peut diviser les anomalies en deux grandes catégories. Dans la première figurent les individus " aveugles " pour le rouge 
ou pour le vert, qui sont dichromates; on peut la subdiviser en deux sous-groupes, selon que fait défaut la vision du rouge (protanopie) ou celle du vert (deutéranopie). La deuxième catégorie comprend les sujets qui voient les trois couleurs fondamentales (trichromates) mais chez lesquels l'une d'elles est mal perçue ou déformée. Des méthodes de comparaison de couleurs permettent de bien analyser les diverses catégories.

Nathans et coll. [2] ont étudié par la méthode de Southern l'ADN de 25 sujets porteurs d'anomalies variées de la vision des couleurs. Les sondes utilisées sont spécifiques des gènes "verts" et "rouges" situés sur le chromosome $\mathrm{X}$. Elles fournissent quatre fragments de restriction appelés $\mathrm{A}, \mathrm{B}, \mathrm{C}$ et $\mathrm{D}$, dont les trois premiers analysent la partie initiale et le quatrième la partie terminale du gène. Comme la longueur de chacun de ces fragments diffère selon qu'il appartient au gène vert ou rouge, on peut les assigner facilement à l'un de ces gènes. Les sujets se divisent en 15 dichromates, chez lesquels manque la vision du rouge $R$ (red) (9 cas) ou celle du vert $G$ (green) (6 cas), et Io trichromates. Deux types de mécanismes moléculaires principaux ont été observés. Le plus rare (6 cas) consiste en la délétion d'un gène; comme nous l'avons montré plus haut, il s'agit toujours du gène $\mathrm{G}$. Le plus fréquent est la présence d'un gène hybride comportant à la fois des éléments $G$ et R. La partie fonctionnellement importante est le segment D correspondant à l'exon 5. Quand la partie proximale (dite $5^{\prime}$ ) du gène est "verte" et la partie distale (dite $3^{\prime}$ ) est "rouge", c'est la vision du vert qui est supprimée ou altérée et vice versa. Il est probable que c'est la position du point de raccord des deux gènes qui détermine la disparition complète ou l'altération d'une couleur. Un modèle plus détaillé est représenté sur la figure. Enfin, un seul sujet a montré une structure apparemment normale des gènes et présente probablement une mutation ponctuelle.

Le nombre de sujets victimes d'anomalies de la vision des couleurs est $m / s n^{\circ} 7$, vol. 2, septembre 86

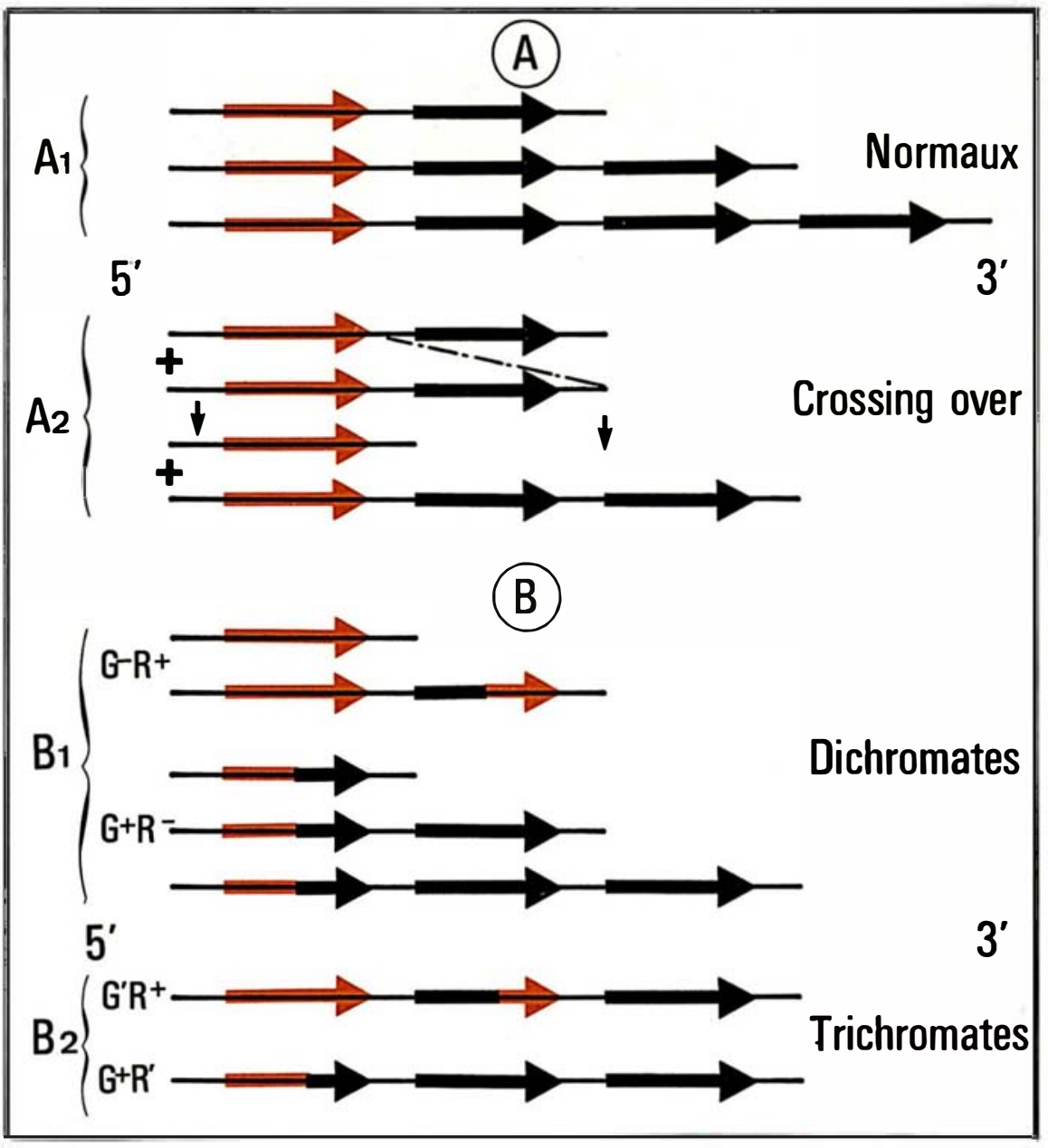

Figure 1.A. Représentation des gènes "vert" et "rouge ". G=vert (green); $R=$ rouge (red).

$A_{l}$. Situation normale: gènes $R$ et $G$ en tandem "tête-queue "; un seul gène $R$; un à trois gènes $G$.

$A_{2}$, Crossing over entre deux allèles au niveau du gène $G$. II en résulte un chromosome sans gène $G$ et un chromosome à deux gènes $G$.

\section{B. Mécanismes moléculaires du daltonisme.}

$B_{1}$. Dichromates

$G^{-} R^{+}$

soit délétion de $G$ résultant du crossing over décrit en $A_{2}$, soit gène hybride 5'G- 3' $R$.

$G^{+} R^{-}$

gène hybride $5^{\prime} R-3^{\prime} G$ suivi ou non de un ou plusieurs gènes $G$ normaux.

$B_{2}$. Trichromates. Le gène anormal est marqué par le signe ' ( $G^{\prime}$ ou $\left.R^{\prime}\right)$

$G^{\prime} R^{+}$

$R$ normal suivi d'un hybride $5^{\prime} R-3^{\prime} G$ suivi lui-même ou non d'un ou deux gènes $G$ normaux.

$G^{+} R^{\prime}$

Hybride $5^{\prime} R-3^{\prime} G$ suivi de gènes $G$ normaux.

On a figuré (ce n'est qu'une hypothèse) la partie $3^{\prime}$ de l'hybride plus courte pour les tri-que pour les dichromates.

Chez les dichromates une des couleurs n'est pas perçue. Chez les trichromates elle est perçue mais la longueur d'onde d'absorption maximum est modifiée. 
très élevé, puisqu'on l'estime à $8 \%$ de la population masculine blanche. Parmi eux environ $30 \%$ sont dichromates, avec un taux similaire d'absence de vision du vert ou du rouge. Sur les $70 \%$ qui restent, on compte un peu plus de $55 \%$ d'anomalies du vert pour moins de $15 \%$ d'anomalies du rouge. On arrive donc à la conclusion surprenante que, d'après les résultats de Nathans et coll., $6 \%$ de la population seraient porteurs d'un gène hybride. Reste à comprendre le mécanisme de formation de cet hybride. Les expériences de Nathans et coll. semblent mettre en jeu soit des recombinaisons non équationnelles, soit des conversions géniques (voir médecine/sciences $n^{\circ}$, vol. I, p. 214). La possibilité d'une mutation ponctuelle, jadis considérée comme la plus probable, n'a été reconnue qu'une fois sur les 25 sujets examinés.

Les travaux de Nathans et coll. ouvrent enfin des perspectives intéressantes du point de vue de l'évolution. L'existence de trois variétés de cônes, porteurs chacun d'un seul type de pigment, est relativement récente. On l'observe chez l'homme, les anthropoïdes et les singes de l'Ancien Monde, mais les singes du Nouveau Monde n'en ont que deux. La séparation des deux premiers pigments remonte au moins à 500 millions d'années, alors que celle des pigments vert et rouge ne date que d'environ 30 millions d'années. Etant données les similitudes de ces deux derniers, une nouvelle duplication pourrait se produire à la suite d'un crossing over propice. Notre vision s'affinera peut-être encore dans l'avenir, si les hommes sont assez patients pour survivre quelques millions d'années...

I. Nathans J, Thomas D, Hogness DS. Molecular genetics of human color vision: the genes encoding bluc, green, and red pigments. Science 1986; 232: 193-202.

2. Nathans J, Piantanida TP, Eddy RL, Shows TB, Hogness DS. Molecular genetics of inherited variation in human color vision. Science 1986; 232: 303-10.

3. Botstein D. The molecular biology of color vision. Science 1986; 232: 142-3.

4. Mollon JD. Understanding colour vision.

\section{Un messager du vieillissement?}

La capacité proliférative des fibroblastes humains en culture est limitée Cela signifie que les cellules vont se diviser un certain nombre de fois (de l'ordre de 50 fois pour des fibroblastes embryonnaires, beaucoup moins pour des fibroblastes prélevés chez des adultes et pratiquement pas pour des fibroblastes de sujets âgés), puis que les divisions vont s'espacer et cesser, les cellules finissant par mourir.

Le phénotype "sénescent ", caractérisé par l'épuisement de la capacité proliférative, semble dominant sur le phénotype " jeune ", voire même sur le phénotype "transformé "* : lorsque des fibroblastes sénescents sont fusionnés avec des cellules jeunes ou avec certaines cellules immortelles, c'est le premier caractère qui l'emporte, et les hybrides ainsi obtenus (dénommés hétérocaryons car ils contiennent plusieurs types de noyaux) ne se divisent pas [r]. Les fibroblastes sénescents sont également résistants à la stimulation de la synthèse d'ADN et de la division cellulaire induite par la microinjection d'oncogènes de type $c$-ras ("transformant "), même associés à un oncogène de type "immortalisant " tel que $E_{1} A$ [2]. L'hypothèse peut donc être faite que s'accumulent dans les fibroblastes, au cours de leur vieillissement, des substances antiprolifératives. Une démonstration directe de ce phénomène vient d'être apportée par Lumpkin et coll. [2]; ces auteurs ont injecté des ARN messagers de cellules sénescentes à des fibroblastes jeunes et démontré qu'il était ainsi possible de bloquer la synthèse d'ADN et la division des cellules. L'effet inhibiteur sur la synthèse d'ADN est I 50 fois plus fort en utilisant l'ARN de cellules sénescentes que de cellules jeunes et est bloqué par les inhibiteurs de la synthèse protéique, démontrant ainsi que l'action se fait par l'intermédiaire d'une protéine codée par les messagers injectés. Cette existence de gènes exprimés au cours du vieillissement et aboutissant à la synthèse de protéines s'opposant à la prolifération cellulaire évoque la notion "d'antioncogène " déjà présentée dans cette revue à propos des cancers congénitaux avec délétions homozygotes de certaines régions chromosomiques [3]. Une même classe de gènes pourrait avoir, physiologiquement, la fonction de contrôler l'action des oncogènes. Leur délétion aboutirait à des proliférations tumorales et leur hyperfonctionnement, qui pourrait être l'un des mécanismes de base du programme génétique de vieillissement, à la sénescence.

L'accumulation des messagers de ces gènes au cours de la sénescence in vitro devrait permettre leur "clonage moléculaire ", la détermination de la séquence protéique de leurs produits et l'analyse de la structure et de la régulation des gènes eux-mêmes... Peut-être une grande date dans les recherches sur les bases biologiques du vieillissement!

A. $\mathbf{K}$.

I. Peircra-Smith OM, Smith JR. Evidence for the recessive nature of cellular immortality. Science 1983; $221: 964$.

2. Lumpkin CK, Keith McClung J, Peircra-Smith OM, Smith JR. Existence of high abundance antiproliferative mRNAs in senescent human diploid fibroblasts. Science 1986; $232: 391-5$. 3. Junien C. Les antioncogènes. médecine/sciences 1986; $2: 238-45$.

* Le "phénotype transformé " est caractérisé par le pouvoir tumorigène des cellules implantées dans ranimal immuno-déprimé, associé à de nombreuses anomalies en cultures et à des modifications morphologiques. 